\title{
Neutrino spin and spin-flavor oscillations in transversal matter currents with standard and nonstandard interactions
}

\author{
Pavel Pustoshny ${ }^{*}$ \\ Department of Theoretical Physics, Moscow State University, 119991 Moscow, Russia \\ Alexander Studenikin ${ }^{\dagger}$ \\ Department of Theoretical Physics, Moscow State University, 119991 Moscow, Russia, \\ and Joint Institute for Nuclear Research, 141980 Dubna, Russia
}

(Received 2 August 2018; published 21 December 2018)

\begin{abstract}
After a brief history of two known types of neutrino mixing and oscillations, including neutrino spin and spin-flavor oscillations in the transversal magnetic field, we perform a systematic study of a new phenomenon of neutrino spin and spin-flavor oscillations engendered by the transversal matter currents on the bases of the developed quantum treatment of the phenomenon. Possibilities for the resonance amplification of these new types of oscillations by the longitudinal matter currents and longitudinal magnetic fields are analyzed. Neutrino spin-flavor oscillations engendered by the transversal matter currents in the case of nonstandard interactions of neutrinos with background matter are also considered.
\end{abstract}

DOI: 10.1103/PhysRevD.98.113009

\section{INTRODUCTION}

Neutrino mixing and oscillations are no doubt among of the most exciting and intriguing phenomena of the present fundamental physics. Being introduced into the physics of elementary particles on the basis of sufficiently general theoretical principles more than 60 years ago [1], these phenomena over the past few decades not only made it possible to obtain a solution to the problem of solar and atmospheric neutrinos, but also marked the beginning of a campaign into a new physics. There are two principal types of neutrino oscillations: flavor and spin oscillations. The former arise when there is an initial inherent mixing of the mass states of the neutrinos, the latter can occur due to the mixing of neutrinos with different polarizations when the magnetic moment of the particle interacts with an external magnetic field.

In this paper, we consider in detail the new possibility of the appearance of spin and spin-flavor neutrino oscillations engendered by weak interactions of neutrinos with the medium in the case when there are the transversal currents of matter. For the neutrino spin oscillations in this case there is no need neither for a neutrino nonzero

\footnotetext{
*kfrepp@gmail.com

†studenik@srd.sinp.msu.ru
}

Published by the American Physical Society under the terms of the Creative Commons Attribution 4.0 International license. Further distribution of this work must maintain attribution to the author(s) and the published article's title, journal citation, and DOI. Funded by SCOAP ${ }^{3}$. magnetic moment nor for an external magnetic field presence.

For the first time the phenomenon of spin oscillations of neutrinos due to weak interactions with the transversal matter currents and/or the transversal polarization of matter was considered in [2] (this possibility was also mentioned in [3]). The existence of the discussed effect of neutrino spin oscillations engendered by the transversal matter current was confirmed in a series of recent papers [4-8] where its possible impact in astrophysics was also discussed.

Given these circumstances there is an urgent need for a systematic and consistent presentation of the theory of this new phenomenon. Indeed, the discussed neutrino spin (and spin-flavor) oscillations engendered by the transversal matter current is a new type of oscillations that have never been discussed before the publication of the paper [2]. Therefore, it would be useful to recall the main points of the history of the neutrino oscillation phenomena studies.

The paper is organized as follows. After a brief history of neutrino mixing and oscillations, give in Sec. II, we present in Sec. III the semiclassical and then quantum treatment of the neutrino spin oscillations $\nu_{e}^{L} \Leftarrow\left(j_{\perp}\right) \Rightarrow \nu_{e}^{R}$ engendered by the transversal matter currents and derive the corresponding neutrino evolution Hamiltonian. In Sec. IV the probability of the neutrino spin oscillations $\nu_{e}^{L} \Leftarrow$ $\left(j_{\perp}, B_{\perp}\right) \Rightarrow \nu_{e}^{R}$ in the transversal matter current and the constant magnetic field is derived. It is shown that there are possibilities for the resonance amplification of the considered spin oscillations $\nu_{e}^{L} \Leftarrow\left(j_{\perp}\right) \Rightarrow \nu_{e}^{R}$ engendered by the transversal matter current due to: (1) the longitudinal matter 
currents, and (2) the longitudinal magnetic field. In Sec. V the neutrino spin-flavor oscillations $\nu_{e}^{L} \Leftarrow\left(j_{\perp}\right) \Rightarrow \nu_{\mu}^{R}$ engendered by the transversal matter current are considered and the resonance amplification of the corresponding probability is discussed. The neutrino spin-flavor oscillations $\nu_{e}^{L} \Leftarrow\left(j_{\perp}\right) \Rightarrow \nu_{\mu}^{R}$ engendered by the transversal matter current with the nonstandard interactions are considered in Sec. VII.

\section{A BRIEF HISTORY OF NEUTRINO MIXING AND OSCILLATIONS}

Neutrino flavor oscillations in vacuum and matter.-The story of the neutrino mixing and oscillations started with two papers by Bruno Pontecorvo [1,9] where the abovementioned effects have been discussed for the first time. In [1] Pontecorvo has indicated that if the neutrino charge were not conserved then the transition between a neutrino and antineutrino would become possible in vacuum. In [9] Pontecorvo has even directly introduced a phenomenon of neutrino mixing. He has written the following.

"Neutrinos in vacuum can transform themselves into antineutrino and vice versa. This means that neutrino and antineutrino are particle mixtures. So, e.g., a beam of neutral leptons from a reactor which at first consists mainly of antineutrinos will change its composition and at a certain distance $R$ from the reactor will be composed of neutrino and antineutrino in equal quantities."

The paper [9] ends with the following statement.

"Under the above assumptions, effects of transformation of neutrino into antineutrino and vice versa may be unobservable in the laboratory because of large value of $R$, but will certainly occur, at least, on an astronomic scale."

A brief history of neutrino mixing and oscillations can be found in [10]. In 1962, just after the discovery of the second flavor neutrino, the effect of neutrino mixing was discussed in [11] where the fields of the weak neutrinos $\nu_{e}$ and $\nu_{\mu}$ were connected with the neutrinos mass states $\nu_{1}$ and $\nu_{2}$ by the unitary mixing matrix $U$ that can be parametrized by the mixing angle $\theta$ and

$\nu_{e}=\nu_{1} \cos \theta+\nu_{2} \sin \theta, \quad \nu_{\mu}=-\nu_{1} \sin \theta+\nu_{2} \cos \theta$.

The theory of neutrino mixing and oscillations was further developed in $[12,13]$ with actual calculations of neutrino beam evolution. In [14] the effect of neutrino interaction with matter of a constant density on neutrino flavor mixing and oscillations was investigated. The existence of resonant amplification of neutrino mixing (the MSW effect) when a neutrino flux propagates through a medium with varying density was predicted in [15].

The tedious studies, both experimental and theoretical, over the past 60 years has been honored by the Nobel Prize of 2015 awarded to Arthur McDonald and Takaaki Kajita for the discovery of neutrino oscillations, which shows that neutrinos have mass.
Neutrino spin oscillations in magnetic fields.-The straightforward consequence of neutrino nonzero mass is the prediction [16] that neutrinos can have nonzero magnetic moments. Studies of neutrino magnetic moments and the related phenomena attract a reasonable interest in literature. The values of neutrino magnetic moments are constrained in the terrestrial laboratory experiments and in the astrophysical considerations (see, for instance, $[17,18]$ ).

Massive neutrinos participate in electromagnetic interactions. The recent review on this topic is given in [19] (the upgrade can be found in [20]). One of the most important phenomena of nontrivial neutrino electromagnetic interactions is the neutrino magnetic moment precession and the corresponding spin oscillations in the presence of external electromagnetic fields. The later effect has been studied in numerous papers published during several passed decades.

Within this scope the neutrino spin oscillations $\nu^{L} \Leftrightarrow \nu^{R}$ induced by the neutrino magnetic moment interaction with the transversal magnetic field $\mathbf{B}_{\perp}$ was first considered in [21]. Then spin-flavor oscillations $\nu_{e}^{L} \Leftrightarrow \nu_{\mu}^{R}$ in $\mathbf{B}_{\perp}$ in vacuum were discussed in [22], the importance of the matter effect was emphasized in [23]. The effect of the resonant amplification of neutrino spin oscillations in $\mathbf{B}_{\perp}$ in the presence of matter was proposed in [24,25], the impact of the longitudinal magnetic field $\mathbf{B}_{\|}$was discussed in [26]. The neutrino spin oscillations in the presence of constant twisting magnetic field were considered in [27-32].

Recently a new approach to the description of neutrino spin and spin-flavor oscillations in the presence of an arbitrary constant magnetic field have been developed [32-34]. Within the new approach exact quantum stationary states are used for classification of neutrino spin states, rather than the neutrino helicity states that have been used for this purpose within the customary approach in many published papers. Recall that the helicity states are not stationary in the presence of a magnetic field. It has been shown [34], in particular, that in the presence of the transversal magnetic field for a given choice of parameters (the energy and magnetic moments of neutrinos and strength of the magnetic field) the amplitude of the flavor oscillations $\nu_{e}^{L} \Leftrightarrow \nu_{\mu}^{L}$ at the vacuum frequency is modulated by the magnetic field frequency. Similar results on the important influence of the transversal magnetic field on amplitudes of various types of neutrino oscillations were obtained earlier [35] on the basis of the exact solution of the effective equation for neutrino evolution in the presence of a magnetic field and matter, which accounts for four neutrino species corresponding to two different flavor states with positive and negative helicities.

In [36] neutrino spin oscillations were considered in the presence of arbitrary constant electromagnetic fields $F_{\mu \nu}$. Neutrino spin oscillations in the presence of the field of circular and linearly polarized electromagnetic waves and superposition of an electromagnetic wave and constant magnetic field were considered in [37-39]. 
The more general case of neutrino spin evolution in the case when the neutrino is subjected to general types of nonderivative interactions with external scalar $s$, pseudoscalar $\pi$, vector $V_{\mu}$, axial-vector $A_{\mu}$, tensor $T_{\mu \nu}$ and pseudotensor $\Pi_{\mu \nu}$ fields was considered in [40]. From the general neutrino spin evolution equation, obtained in [40], it follows that neither scalar $s$ nor pseudoscalar $\pi$ nor vector $V_{\mu}$ fields can induce neutrino spin evolution. On the contrary, within the general consideration of neutrino spin evolution it was shown that electromagnetic (tensor) and weak (axial-vector) interactions can contribute to the neutrino spin evolution.

Recently we have considered in detail $[33,41]$ neutrino mixing and oscillations in the arbitrary constant magnetic field that have $\mathbf{B}_{\perp}$ and $\mathbf{B}_{\|}$nonzero components and derived an explicit expressions for the effective neutrino magnetic moments for the flavor neutrinos in terms of the corresponding magnetic moments introduced in the neutrino mass basis.

\section{NEUTRINO SPIN OSCILLATIONS $\nu_{e}^{L} \Leftarrow\left(j_{\perp}\right) \Rightarrow \nu_{e}^{R}$ ENGENDERED BY TRANSVERSAL MATTER CURRENTS}

For many years, until 2004, it was believed that a neutrino helicity precession and the corresponding spin oscillations can be induced by the neutrino magnetic interactions with an external electromagnetic field that provided the existence of the transversal magnetic field component $\mathbf{B}_{\perp}$ in the particles rest frame. A new and very interesting possibility for neutrino spin (and spin-flavor) oscillations engendered by the neutrino interaction with matter background was proposed and investigated for the first time in [2]. It was shown [2] that neutrino spin oscillations can be induced not only by the neutrino interaction with a magnetic field, as it was believed before, but also by neutrino interactions with matter in the case when there is a transversal matter current or matter polarization. This new effect has been explicitly highlighted in [2].

"The possible emergence of neutrino spin oscillations owing to neutrino interaction with matter under the condition that there exists a nonzero transverse current component or matter polarization is the most important new effect that follows from the investigation of neutrino-spin oscillations in Sec. IV. So far, it has been assumed that neutrino-spin oscillations may arise only in the case where there exists a nonzero transverse magnetic field in the neutrino rest frame."

For historical notes reviewing studies of the discussed effect, see in $[42,43]$. It should be noted that the predicted effect exists regardless of the composition of the background matter transversal current and the source of its possible polarization (that can be a background magnetic field, for instance).
Note that the existence of the discussed effect of neutrino spin oscillations engendered by the transversal matter current and its possible impact in astrophysics was confirmed in a series of recent papers [4-7]. In the most recent paper [8] it has been pointed out that the effect of neutrino spin conversion from left-handed helicity states to righthanded helicity states in the absence of a magnetic field or a large magnetic moment (that was first predicted in [2]) would be present in a supernova environment.

\section{A. Semiclassical treatment}

Following the discussion in [2] consider, as an example, an electron neutrino spin precession in the case when neutrinos with the Standard Model interaction are propagating through moving and polarized matter composed of electrons (electron gas) in the presence of an electromagnetic field given by the electromagnetic-field tensor $F_{\mu \nu}=(\mathbf{E}, \mathbf{B})$. To derive the neutrino spin oscillation probability in the transversal matter current we use the generalized BargmannMichel-Telegdi equation that describes the evolution of the three-dimensional neutrino spin vector $\mathbf{S}$,

$$
\frac{d \mathbf{S}}{d t}=\frac{2}{\gamma}\left[\boldsymbol{S} \times\left(\boldsymbol{B}_{0}+\boldsymbol{M}_{0}\right)\right],
$$

where the magnetic field $\mathbf{B}_{0}$ in the neutrino rest frame is determined by the transversal and longitudinal (with respect to the neutrino motion) magnetic and electric field components in the laboratory frame,

$$
\mathbf{B}_{0}=\gamma\left(\mathbf{B}_{\perp}+\frac{1}{\gamma} \mathbf{B}_{\|}+\sqrt{1-\gamma^{-2}}\left[\mathbf{E}_{\perp} \times \frac{\boldsymbol{\beta}}{\beta}\right]\right),
$$

$\gamma=\left(1-\beta^{2}\right)^{-\frac{1}{2}}, \boldsymbol{\beta}$ is the neutrino velocity. The matter term $\mathbf{M}_{0}$ in Eq. (2) is also composed of the transversal $\mathbf{M}_{0_{\|}}$and longitudinal $\mathbf{M}_{0_{\perp}}$ parts,

$$
\begin{aligned}
\mathbf{M}_{0}= & \mathbf{M}_{0_{\|}}+\mathbf{M}_{0_{\perp}}, \\
\mathbf{M}_{0_{\|}}= & \gamma \boldsymbol{\beta} \frac{n_{0}}{\sqrt{1-v_{e}^{2}}}\left\{\rho_{e}^{(1)}\left(1-\frac{\mathbf{v}_{e} \boldsymbol{\beta}}{1-\gamma^{-2}}\right)\right\} \\
& -\frac{\rho_{e}^{(2)}}{1-\gamma^{-2}}\left\{\boldsymbol{\zeta}_{e} \boldsymbol{\beta} \sqrt{1-v_{e}^{2}}+\left(\boldsymbol{\zeta}_{e} \mathbf{v}_{e} \frac{\left(\boldsymbol{\beta} \mathbf{v}_{e}\right)}{1+\sqrt{1-v_{e}^{2}}}\right)\right\},
\end{aligned}
$$

$$
\begin{aligned}
\mathbf{M}_{0_{\perp}}= & -\frac{n_{0}}{\sqrt{1-v_{e}^{2}}}\left\{\mathbf{v}_{e_{\perp}}\left(\rho_{e}^{(1)}+\rho_{e}^{(2)} \frac{\left(\boldsymbol{\zeta}_{e} \mathbf{v}_{e}\right)}{1+\sqrt{1-v_{e}^{2}}}\right)\right. \\
& \left.+\zeta_{e_{\perp}} \rho_{e}^{(2)} \sqrt{1-v_{e}^{2}}\right\}
\end{aligned}
$$

Here $n_{0}=n_{e} \sqrt{1-v_{e}^{2}}$ is the invariant number density of matter given in the reference frame for which the total 
speed of matter is zero. The vectors $\mathbf{v}_{e}$ and $\zeta_{e}\left(0 \leq\left|\boldsymbol{\zeta}_{e}\right|^{2} \leq 1\right)$ denote, respectively, the speed of the reference frame in which the mean momentum of matter (electrons) is zero, and the mean value of the polarization vector of the background electrons in the above-mentioned reference frame. The coefficients $\rho_{e}^{(1,2)}$ calculated within the extended Standard Model supplied with $S U(2)$-singlet right-handed neutrino $\nu_{R}$ are, respectively, $\rho_{e}^{(1)}=\frac{\tilde{G}_{F}}{2 \sqrt{2} \mu}$ and $\rho_{e}^{(2)}=-\frac{G_{F}}{2 \sqrt{2} \mu}$, where $\tilde{G}_{F}=G_{F}\left(1+4 \sin ^{2} \theta_{W}\right)$.

For neutrino evolution between two neutrino states $\nu_{e}^{L} \Leftrightarrow \nu_{e}^{R}$ in the presence of the magnetic field and moving matter we get [2] the following equation:

$i \frac{d}{d t}\left(\begin{array}{c}\nu_{e}^{L} \\ \nu_{e}^{R}\end{array}\right)=\mu\left(\begin{array}{cc}\frac{1}{\gamma}\left|\mathbf{M}_{0 \|}+\mathbf{B}_{0 \|}\right| & \left|\mathbf{B}_{\perp}+\frac{1}{\gamma} \mathbf{M}_{0 \perp}\right| \\ \left|\mathbf{B}_{\perp}+\frac{1}{\gamma} \mathbf{M}_{0 \perp}\right| & -\frac{1}{\gamma}\left|\mathbf{M}_{0 \|}+\mathbf{B}_{0 \|}\right|\end{array}\right)\left(\begin{array}{c}\nu_{e}^{L} \\ \nu_{e}^{R}\end{array}\right)$.

Thus, the probability of the neutrino spin oscillations in the adiabatic approximation is given by [2]

$$
\begin{aligned}
P_{\nu_{e}^{L} \rightarrow \nu_{e}^{R}}(x) & =\sin ^{2} 2 \theta_{\mathrm{eff}} \sin ^{2} \frac{\pi x}{L_{\mathrm{eff}}}, \\
\sin ^{2} 2 \theta_{\mathrm{eff}} & =\frac{E_{\mathrm{eff}}^{2}}{E_{\mathrm{eff}}^{2}+\Delta_{\mathrm{eff}}^{2}}, \\
L_{\mathrm{eff}} & =\frac{\pi}{\sqrt{E_{\mathrm{eff}}^{2}+\Delta_{\mathrm{eff}}^{2}}},
\end{aligned}
$$

where

$$
\begin{aligned}
& E_{\text {eff }}=\mu\left|\mathbf{B}_{\perp}+\frac{1}{\gamma} \mathbf{M}_{0 \perp}\right|, \\
& \Delta_{\text {eff }}=\frac{\mu}{\gamma}\left|\mathbf{M}_{0 \|}+\mathbf{B}_{0 \|}\right| .
\end{aligned}
$$

Thus, it follows that even without the presence of an electromagnetic field, $\mathbf{B}_{\perp}=\mathbf{B}_{0 \|}=0$, neutrino spin oscillations $\nu_{e}^{L} \Leftrightarrow \nu_{e}^{R}$ can be induced in the presence of matter when the transverse matter term $\mathbf{M}_{0 \perp}$ is not zero. If we neglect possible effects of matter polarization then the neutrino evolution equation (7) simplifies to

$$
i \frac{d}{d t}\left(\begin{array}{c}
\nu_{e}^{L} \\
\nu_{e}^{R}
\end{array}\right)=\frac{\mu}{\gamma}\left(\begin{array}{cc}
M_{0 \|} & M_{0 \perp} \\
M_{0 \perp} & -M_{0 \|}
\end{array}\right)\left(\begin{array}{c}
\nu_{e}^{L} \\
\nu_{e}^{R}
\end{array}\right)
$$

where

$$
\begin{aligned}
& \mathbf{M}_{0_{\|}}=\gamma \boldsymbol{\beta} \rho_{e}^{(1)}\left(1-\frac{\mathbf{v}_{e} \boldsymbol{\beta}}{1-\gamma^{-2}}\right) \frac{n_{0}}{\sqrt{1-v_{e}^{2}}}, \\
& \mathbf{M}_{0_{\perp}}=-\rho_{e}^{(1)} \mathbf{v}_{e_{\perp}} \frac{n_{0}}{\sqrt{1-v_{e}^{2}}} .
\end{aligned}
$$

The effective mixing angle and oscillation length in the neutrino spin oscillation probability (8) now are given by

$$
\sin ^{2} 2 \theta_{\mathrm{eff}}=\frac{M_{0 \perp}^{2}}{M_{0 \|}^{2}+M_{0 \perp}^{2}}, \quad L_{\mathrm{eff}}=\frac{\pi}{\mu M_{0}} \gamma
$$

The above considerations can be applied to other types of neutrinos and various matter compositions. It is also obvious that for neutrinos with nonzero transition magnetic moments a similar effect for spin-flavor oscillations exists under the same background conditions.

\section{B. Quantum treatment}

Here below we continue our studies of the effect of neutrino spin evolution induced by the transversal matter currents and develop a consistent derivation (see also $[44,45])$ of the effect based on the direct calculation of the spin evolution effective Hamiltonian in the case when a neutrino is propagating in the transversal currents of matter.

Consider two flavor neutrinos with two possible helicities $\nu_{f}=\left(\nu_{e}^{+}, \nu_{e}^{-}, \nu_{\mu}^{+}, \nu_{\mu}^{-}\right)^{T}$ in moving matter composed of neutrons. The neutrino interaction Lagrangian reads

$$
\begin{aligned}
L_{\mathrm{int}} & =-f^{\mu} \sum_{l} \bar{\nu}_{l}(x) \gamma_{\mu} \frac{1+\gamma_{5}}{2} \nu_{l}(x) \\
& =-f^{\mu} \sum_{i} \bar{\nu}_{i}(x) \gamma_{\mu} \frac{1+\gamma_{5}}{2} \nu_{i}(x), \\
f^{\mu} & =-\frac{G_{F}}{\sqrt{2}} n(1, \mathbf{v}),
\end{aligned}
$$

where $l=e$, or $\mu$ indicates the neutrino flavor, $i=1,2$ indicates the neutrino mass state and the matter potential $f^{\mu}$ depends on the neutron number density in the laboratory reference frame $n=\frac{n_{0}}{\sqrt{1-v^{2}}}$ and on the velocity of matter $\mathbf{v}=\left(v_{1}, v_{2}, v_{3}\right)$. Each of the flavor neutrinos is a superposition of the neutrino mass states,

$$
\begin{aligned}
& \nu_{e}^{ \pm}=\nu_{1}^{ \pm} \cos \theta+\nu_{2}^{ \pm} \sin \theta, \\
& \nu_{\mu}^{ \pm}=-\nu_{1}^{ \pm} \sin \theta+\nu_{2}^{ \pm} \cos \theta .
\end{aligned}
$$

The neutrino evolution equation in the flavor basis is

$$
i \frac{d}{d t} \nu_{f}=\left(H_{0}+\Delta H_{\mathrm{SM}}\right) \nu_{f},
$$

where the first term $H_{0}$ of the effective Hamiltonian determines the neutrino evolution in nonmoving matter. The second term $\Delta H_{\mathrm{SM}}$ accounts for the effect of matter motion and it can be expressed as (see also $[44,45]$ ) 


$$
\Delta H_{\mathrm{SM}}=\left(\begin{array}{cccc}
\Delta_{e e}^{++} & \Delta_{e e}^{+-} & \Delta_{e \mu}^{++} & \Delta_{e \mu}^{+-} \\
\Delta_{e e}^{-+} & \Delta_{e e}^{--} & \Delta_{e \mu}^{-+} & \Delta_{e \mu}^{--} \\
\Delta_{\mu e}^{++} & \Delta_{\mu e}^{+-} & \Delta_{\mu \mu}^{++} & \Delta_{\mu \mu}^{+-} \\
\Delta_{\mu e}^{-+} & \Delta_{\mu e}^{--} & \Delta_{\mu \mu}^{-+} & \Delta_{\mu \mu}^{--}
\end{array}\right),
$$

where

$$
\Delta_{k l}^{s s^{\prime}}=\left\langle\nu_{k}^{s}\left|\Delta H_{\mathrm{SM}}\right| \nu_{l}^{s^{\prime}}\right\rangle, \quad k, l=e, \mu, \quad s, s^{\prime}= \pm .
$$

From (13) it follows that

$$
\begin{aligned}
\Delta H_{\mathrm{SM}} & =\frac{G_{F}}{2 \sqrt{2}} n\left(1+\gamma_{5}\right) \mathbf{v} \boldsymbol{\gamma}, \\
\mathbf{v} \boldsymbol{\gamma} & =v_{1} \gamma_{1}+v_{2} \gamma_{2}+v_{3} \gamma_{3} .
\end{aligned}
$$

In evaluation of $\Delta_{k l}^{s s^{\prime}}$ we have first introduced the neutrino flavor states $\nu_{k}^{s}$ and $\nu_{l}^{s^{\prime}}$ as superpositions of the mass states $\nu_{1,2}^{ \pm}$. Then, using the exact free neutrino mass states spinors,

$$
\nu_{\alpha}^{s}=C_{\alpha} \sqrt{\frac{E_{\alpha}+m_{\alpha}}{2 E_{\alpha}}}\left(\begin{array}{c}
u_{\alpha}^{s} \\
\frac{\sigma p_{\alpha}}{E_{\alpha}+m_{\alpha}} u_{\alpha}^{s}
\end{array}\right) e^{i p_{\alpha} x}, \quad \alpha=1,2,
$$

where the two-component spinors $u_{\alpha}^{s}$,

$$
u_{\alpha}^{s=1}=\left(\begin{array}{l}
1 \\
0
\end{array}\right), \quad u_{\alpha}^{s=-1}=\left(\begin{array}{l}
0 \\
1
\end{array}\right),
$$

define neutrino helicity states, we have performed calculations that are analogous to those performed in [41]. The difference in calculations is that here we consider not electromagnetic neutrino interaction with a magnetic field but the neutrino weak interaction with moving matter given by (16). For the typical term $\Delta_{\alpha \alpha^{\prime}}^{s s^{\prime}}=\left\langle\nu_{\alpha}^{s}\left|\Delta H_{\mathrm{SM}}\right| \nu_{\alpha^{\prime}}^{s^{\prime}}\right\rangle$, which by fixing proper values of $\alpha, s, \alpha^{\prime}$ and $s^{\prime}$ can reproduce all of the elements of the neutrino evolution Hamiltonian $\Delta H^{\text {eff }}$ that accounts for the effect of matter motion, we obtain (see also $[44,45])$

$$
\begin{aligned}
\Delta_{\alpha \alpha^{\prime}}^{s s^{\prime}}= & \frac{G_{F}}{2 \sqrt{2}} \frac{n_{0}}{\sqrt{1-v^{2}}}\left\{u _ { \alpha } ^ { s T } \left[\left(1-\sigma_{3}\right) v_{\|}\right.\right. \\
& \left.\left.+\left(\gamma_{\alpha \alpha^{\prime}}{ }^{-1} \sigma_{1}+i \tilde{\gamma}_{\alpha \alpha^{\prime}}{ }^{-1} \sigma_{2}\right) v_{\perp}\right] u_{\alpha^{\prime}}^{s^{\prime}}\right\} \delta_{\alpha}^{\alpha^{\prime}},
\end{aligned}
$$

where $v_{\|}$and $v_{\perp}$ are the longitudinal and transversal velocities of the matter current and

$$
\begin{aligned}
\gamma_{\alpha \alpha^{\prime}}{ }^{-1} & =\frac{1}{2}\left(\gamma_{\alpha}^{-1}+\gamma_{\alpha^{\prime}}^{-1}\right), \\
\tilde{\gamma}_{\alpha \alpha^{\prime}}{ }^{-1} & =\frac{1}{2}\left(\gamma_{\alpha}^{-1}-\gamma_{\alpha^{\prime}}^{-1}\right), \\
\gamma_{\alpha}^{-1} & =\frac{m_{\alpha}}{E_{\alpha}} .
\end{aligned}
$$

Recalling expressions for the Pauli matrixes,

$$
\sigma_{3}=\left(\begin{array}{cc}
1 & 0 \\
0 & -1
\end{array}\right), \quad \sigma_{1}=\left(\begin{array}{ll}
0 & 1 \\
1 & 0
\end{array}\right), \quad \sigma_{2}=i\left(\begin{array}{cc}
0 & -1 \\
1 & 0
\end{array}\right),
$$

we get (see also $[44,45])$

$$
\begin{aligned}
\Delta_{\alpha \alpha^{\prime}}^{s s^{\prime}} & =\frac{G_{F}}{2 \sqrt{2}} \frac{n_{0}}{\sqrt{1-v^{2}}} \\
& \times\left\{u_{\alpha}^{s T}\left[\left(\begin{array}{ll}
0 & 0 \\
0 & 2
\end{array}\right) v_{\|}+\left(\begin{array}{cc}
0 & \gamma_{\alpha}^{-1} \\
\gamma_{\alpha^{\prime}}^{-1} & 0
\end{array}\right) v_{\perp}\right] u_{\alpha^{\prime}}^{s^{\prime}}\right\} \delta_{\alpha}^{\alpha^{\prime}} .
\end{aligned}
$$

The obtained general expression (24) can be used for investigations of various types of neutrino spin oscillations in the transversal matter currents considered in the neutrino mass basis. It confirms our prediction [2] that there are the effect of the neutrino spin conversion and corresponding spin oscillations engendered by the interaction with the transversal current of matter. It is also clear that the corresponding effect engendered by the transversal polarization of matter can be treated in much the same way.

On the basis of Eq. (24) and using the relation (14) between neutrino mass $\nu_{\alpha}^{ \pm}$and flavor $\nu_{l}^{ \pm}$states it is possible to bring our considerations to observational terms and study neutrino oscillations in the flavor basis $\nu_{f}^{s}$. The neutrino flavor and mass states are connected by the neutrino mixing matrix,

$$
\nu_{f}=U \nu_{\alpha}
$$

for which in the considered case we have

$$
U=\left(\begin{array}{cccc}
\cos \theta & 0 & \sin \theta & 0 \\
0 & \cos \theta & 0 & \sin \theta \\
-\sin \theta & 0 & \cos \theta & 0 \\
0 & -\sin \theta & 0 & \cos \theta
\end{array}\right) .
$$

The corresponding neutrino evolution equation is

$$
i \frac{d}{d t} \nu_{f}=H_{v}^{f} \nu_{f},
$$

where the effective Hamiltonian is given by $H_{v}^{f}=U H U^{\dagger}$ and can be directly calculated using Eq. (26).

However, it is possible to get a general structure of the effective evolution Hamiltonian for the flavor neutrino using results of our previous studies $[33,41]$ of neutrino oscillations in the arbitrary magnetic field $\boldsymbol{B}=\boldsymbol{B}_{\|}+\boldsymbol{B}_{\perp}$. For evaluation of the flavor neutrino oscillation in an arbitrary moving matter that is characterized by the current $\boldsymbol{j}=$ $\boldsymbol{j}_{\|}+\boldsymbol{j}_{\perp}$ we consider results for the flavor neutrino oscillations in the magnetic field $\boldsymbol{B}$ and account for similarity of the 
correspondence between the neutrino magnetic moment to magnetic field interaction Hamiltonian $H_{B}$,

$H_{B}=-\mu_{\alpha \alpha^{\prime}} \bar{\nu}_{\alpha^{\prime}} \mathbf{\Sigma} \mathbf{B} \nu_{\alpha}+$ H.c., $\quad \Sigma_{i}=\left(\begin{array}{cc}\sigma_{i} & 0 \\ 0 & \sigma_{i}\end{array}\right)$,

and the neutrino to moving matter interaction Hamiltonian $H_{v}$,

$$
H_{v}=\tilde{G} n \bar{\nu}_{\alpha^{\prime}} \boldsymbol{v} \gamma \nu_{\alpha}, \quad \tilde{G}=\frac{G_{F}}{2 \sqrt{2}} .
$$

Here $n=\frac{n_{0}}{\sqrt{1-v^{2}}}=n_{0} \gamma_{n}, n_{0}$ is the invariant density of matter composed of neutrons. For the flavor neutrino evolution Hamiltonian in the magnetic field $H_{B}^{f}=U H_{B} U^{\dagger}$ we have $[33,41,46]$

$$
H_{B}^{f}=\left(\begin{array}{cccc}
-\left(\frac{\mu}{\gamma}\right)_{e e} B_{\|} & \mu_{e e} B_{\perp} & -\left(\frac{\mu}{\gamma}\right)_{e \mu} B_{\|} & \mu_{e \mu} B_{\perp} \\
\mu_{e e} B_{\perp} & \left(\frac{\mu}{\gamma}\right)_{e e} B_{\|} & \mu_{e \mu} B_{\perp} & \left(\frac{\mu}{\gamma}\right)_{e \mu} B_{\|} \\
-\left(\frac{\mu}{\gamma}\right)_{e \mu} B_{\|} & \mu_{e \mu} B_{\perp} & -\left(\frac{\mu}{\gamma}\right)_{\mu \mu} B_{\|} & \mu_{\mu \mu} B_{\perp} \\
\mu_{e \mu} B_{\perp} & \left(\frac{\mu}{\gamma}\right)_{e \mu} B_{\|} & \mu_{\mu \mu} B_{\perp} & \left(\frac{\mu}{\gamma}\right)_{\mu \mu} B_{\|}
\end{array}\right),
$$

where the following notations are used:

$$
\begin{aligned}
\left(\frac{\mu}{\gamma}\right)_{e e} & =\frac{\mu_{11}}{\gamma_{11}} \cos ^{2} \theta+\frac{\mu_{22}}{\gamma_{22}} \sin ^{2} \theta+\frac{\mu_{12}}{\gamma_{12}} \sin 2 \theta, \\
\left(\frac{\mu}{\gamma}\right)_{e \mu} & =\frac{\mu_{12}}{\gamma_{12}} \cos 2 \theta+\frac{1}{2}\left(\frac{\mu_{22}}{\gamma_{22}}-\frac{\mu_{11}}{\gamma_{11}}\right) \sin 2 \theta, \\
\left(\frac{\mu}{\gamma}\right)_{\mu \mu} & =\frac{\mu_{11}}{\gamma_{11}} \sin ^{2} \theta+\frac{\mu_{22}}{\gamma_{22}} \cos ^{2} \theta-\frac{\mu_{12}}{\gamma_{12}} \sin 2 \theta, \\
\mu_{e e} & =\mu_{11} \cos ^{2} \theta+\mu_{22} \sin ^{2} \theta+\mu_{12} \sin 2 \theta \\
\mu_{e \mu} & =\mu_{12} \cos 2 \theta+\frac{1}{2}\left(\mu_{22}-\mu_{11}\right) \sin 2 \theta, \\
\mu_{\mu \mu} & =\mu_{11} \sin ^{2} \theta+\mu_{22} \cos ^{2} \theta-\mu_{12} \sin 2 \theta .
\end{aligned}
$$

For the flavor neutrino evolution Hamiltonian in moving matter $H_{v}^{f}=U H_{v} U^{\dagger}$ we get
$H_{v}^{f}=n \tilde{G}\left(\begin{array}{cccc}0 & \left(\frac{\eta}{\gamma}\right)_{e e} v_{\perp} & 0 & \left(\frac{\eta}{\gamma}\right)_{e \mu} v_{\perp} \\ \left(\frac{\eta}{\gamma}\right)_{e e} v_{\perp} & 2 \eta_{e e}\left(1-v_{\|}\right) & \left(\frac{\eta}{\gamma}\right)_{e \mu} v_{\perp} & \eta_{e \mu} \\ 0 & \left(\frac{\eta}{\gamma}\right)_{e \mu} v_{\perp} & 0 & \left(\frac{\eta}{\gamma}\right)_{\mu \mu} v_{\perp} \\ \left(\frac{\eta}{\gamma}\right)_{e \mu} v_{\perp} & \eta_{e \mu} & \left(\frac{\eta}{\gamma}\right)_{\mu \mu} v_{\perp} & 2 \eta_{\mu \mu}\left(1-v_{\|}\right)\end{array}\right)$,

where $\frac{\eta}{\gamma}$ and $\eta$ are given by

$$
\begin{gathered}
\left(\frac{\eta}{\gamma}\right)_{e e}=\left(\frac{\mu}{\gamma}\right)_{e e_{\mid \mu_{11}=\mu_{22}=1, \mu_{12}=0}}=\frac{\cos ^{2} \theta}{\gamma_{11}}+\frac{\sin ^{2} \theta}{\gamma_{22}}, \\
\left(\frac{\eta}{\gamma}\right)_{\mu \mu}=\left(\frac{\mu}{\gamma}\right)_{\mu \mu_{\mid \mu_{11}=\mu_{22}=1, \mu_{12}=0}}=\frac{\sin ^{2} \theta}{\gamma_{11}}+\frac{\cos ^{2} \theta}{\gamma_{22}}, \\
\left(\frac{\eta}{\gamma}\right)_{e \mu}=\left(\frac{\mu}{\gamma}\right)_{e \mu_{\mid \mu_{11}=\mu_{22}=1, \mu_{12}=0}}=\frac{\sin 2 \theta}{\tilde{\gamma}_{21}}, \\
\eta_{e e}=\mu_{e e_{\mid \mu_{11}=\mu_{22}=1, \mu_{12}=0}=1,} \\
\eta_{\mu \mu}=\mu_{\mu \mu_{\mid \mu_{11}=\mu_{22}=1, \mu_{12}=0}}=1, \\
\eta_{e \mu}=\mu_{e \mu_{\mid \mu_{11}=\mu_{22}=1, \mu_{12}=0}=0}=0
\end{gathered}
$$

if one sets $\mu_{11}=\mu_{22}=1, \mu_{12}=0$ in Eqs. (31) and (32).

From the above consideration it follows that neutrino spin oscillations can be engendered by the magnetic moment interactions with the transversal magnetic field $\boldsymbol{B}_{\perp}$ as well as by the neutrino weak interactions with the transversal matter current $\boldsymbol{j}_{\perp}$.

\section{PROBABILITY OF NEUTRINO SPIN OSCILLATIONS $\nu_{e}^{L} \Leftarrow\left(\boldsymbol{j}_{\perp}, B_{\perp}\right) \Rightarrow \nu_{e}^{R}$ ENGENDERED BY TRANSVERSAL MATTER CURRENT AND MAGNETIC FIELD}

Consider the initial neutrino state $\nu_{e}^{L}$ moving in the background with the magnetic field $\boldsymbol{B}=\boldsymbol{B}_{\|}+\boldsymbol{B}_{\perp}$ and nonzero matter current $\boldsymbol{j}=\boldsymbol{j}_{\|}+\boldsymbol{j}_{\perp}$. One of the possible modes of neutrino transitions with the change of helicity is $\nu_{e}^{L} \Leftarrow\left(j_{\perp}, B_{\perp}\right) \Rightarrow \nu_{e}^{R}$. The corresponding oscillations are governed by the evolution equation

$$
i \frac{d}{d t}\left(\begin{array}{c}
\nu_{e}^{L} \\
\nu_{e}^{R}
\end{array}\right)=\left(\begin{array}{cc}
\left(\frac{\mu}{\gamma}\right)_{e e} B_{\|}+\eta_{e e} \tilde{G} n(1-\boldsymbol{v} \boldsymbol{\beta}) & \mu_{e e} B_{\perp}+\left(\frac{\eta}{\gamma}\right)_{e e} \tilde{G} n v_{\perp} \\
\mu_{e e} B_{\perp}+\left(\frac{\eta}{\gamma}\right)_{e e} \tilde{G} n v_{\perp} & -\left(\frac{\mu}{\gamma}\right)_{e e} B_{\|}-\eta_{e e} \tilde{G} n(1-\boldsymbol{\gamma} \boldsymbol{\beta})
\end{array}\right)\left(\begin{array}{c}
\nu_{e}^{L} \\
\nu_{e}^{R}
\end{array}\right) .
$$


For the oscillation $\nu_{e}^{L} \Leftarrow\left(j_{\perp}, B_{\perp}\right) \Rightarrow \nu_{e}^{R}$ probability we get

$$
\begin{aligned}
P_{\nu_{e}^{L} \rightarrow \nu_{e}^{R}}(x) & =\frac{E_{\mathrm{eff}}^{2}}{E_{\mathrm{eff}}^{2}+\Delta_{\mathrm{eff}}^{2}} \sin ^{2} \frac{\pi x}{L_{\mathrm{eff}}}, \\
L_{\mathrm{eff}} & =\frac{\pi}{\sqrt{E_{\mathrm{eff}}^{2}+\Delta_{\mathrm{eff}}^{2}}},
\end{aligned}
$$

where

$$
\begin{aligned}
& E_{\mathrm{eff}}=\left|\mu_{e e} \boldsymbol{B}_{\perp}+\left(\frac{\eta}{\gamma}\right)_{e e} \tilde{G} n \boldsymbol{v}_{\perp}\right|, \\
& \Delta_{\mathrm{eff}}=\left|\left(\frac{\mu}{\gamma}\right)_{e e} \boldsymbol{B}_{\|}+\eta_{e e} \tilde{G} n(1-\boldsymbol{v} \boldsymbol{\beta}) \boldsymbol{\beta}\right| .
\end{aligned}
$$

In the next section we estimate values of the corresponding parameters that characterize the properties of neutrinos, the background matter and the magnetic field for which neutrino spin oscillations $\nu_{e}^{L} \Leftarrow\left(j_{\perp}\right) \Rightarrow \nu_{e}^{R}$, engendered by neutrino weak interactions with the transversal matter current, can proceed with significant probability.

\section{A. Resonance amplification of neutrino spin oscillations $\nu_{e}^{L} \Leftarrow\left(j_{\perp}\right) \Rightarrow \nu_{e}^{R}$ by longitudinal matter current}

We are interested in the situation when the amplitude of oscillations $\sin ^{2} 2 \theta_{\text {eff }}$ in (41) is not small and we use the criterion based on the demand that

$$
\sin ^{2} 2 \theta_{\text {eff }}=\frac{E_{\text {eff }}^{2}}{E_{\text {eff }}^{2}+\Delta_{\text {eff }}^{2}} \geq \frac{1}{2},
$$

which is provided by the condition $E_{\text {eff }} \geq \Delta_{\text {eff }}$.

At first we consider the case when the effect of magnetic field is negligible and thus we have

$$
E_{\mathrm{eff}}=\left|\left(\frac{\eta}{\gamma}\right)_{e e} \tilde{G} n \boldsymbol{v}_{\perp}\right|, \quad \Delta_{\mathrm{eff}}=|\tilde{G} n(1-\boldsymbol{v} \boldsymbol{\beta}) \boldsymbol{\beta}|
$$

and the oscillation length is given by

$$
L_{\mathrm{eff}}=\frac{\pi}{\left(\frac{\eta}{\gamma}\right)_{e e} \tilde{G} n v_{\perp}} .
$$

From the condition $E_{\text {eff }} \geq \Delta_{\text {eff }}$ it follows that

$$
\left(\frac{\eta}{\gamma}\right)_{e e} v_{\perp} \geq(1-v \boldsymbol{\beta})
$$

In the further evaluations we suppose that $\Delta m=$ $m_{2}-m_{1} \ll m_{1}, m_{2}$, and introduce the neutrino effective gamma-factor $\gamma_{\nu}$ :

$$
\frac{1}{\gamma_{\nu}}=\frac{1}{\gamma_{11}} \sim \frac{1}{\gamma_{22}}
$$

Then the condition (46) reduces to

$$
\frac{v_{\perp}}{\gamma_{\nu}} \geq(1-\boldsymbol{v} \boldsymbol{\beta})
$$

Assuming neutrino masses $m_{1}, m_{2} \sim 0.1 \mathrm{eV}$, for a typical neutrino energy $p_{0}^{\nu} \sim 10 \mathrm{MeV}$ we find $\gamma_{\nu} \sim 10^{7}$. Consider the case when neutrinos are more relativistic particles than the background matter neutrons $\left(\gamma_{\nu} \gg \gamma_{n}\right)$, then from (48) we get

$$
\frac{1}{\gamma_{\nu}} \geq \frac{1}{\gamma_{n}^{2}}
$$

The latter condition can be valid for ultrarelativistic background matter with $\gamma_{n} \geq \gamma_{\nu}{ }^{1 / 2} \sim 3 \times 10^{3}$. At the same time the oscillation length $L_{\text {eff }}$ given by (45) can be $L_{\text {eff }} \sim 50 \mathrm{~km}$ in the case $n \sim 10^{37} \mathrm{~cm}^{3}$ and $\gamma_{n} \sim 3 \times 10^{3}$.

\section{B. Resonance amplification of neutrino spin oscillations $\nu_{e}^{L} \Leftarrow\left(j_{\perp}\right) \Rightarrow \nu_{e}^{R}$ by longitudinal magnetic field}

The presence of the longitudinal magnetic field $\boldsymbol{B}_{\|}$can also have an important impact on the criterion (90). In the previous consideration the diminishing value of $\Delta_{\text {eff }}$ is attained by the vanishing value of $(1-\boldsymbol{v} \boldsymbol{\beta})$. Now we consider reduction of the term $\tilde{G} n(1-\boldsymbol{v} \boldsymbol{\beta})$ in $\Delta_{\text {eff }}$ given by (42) due to the contribution of $\left(\frac{\mu}{\gamma}\right)_{e e} \boldsymbol{B}_{\|}$. This possibility can be realized when $\boldsymbol{B}_{\|} \boldsymbol{\beta}=-1$. An environment we are considering can be realized by models of short gamma-ray bursts (sGRB) (see $[47,48]$ ). Consider the neutrino $\nu_{e}$ escaping the central neutron star with inclination given by an angle $\alpha$ from the plane of the accretion disk. Then this neutrino propagates through the toroidal bulk of very dense matter that rotates with the angular velocity of about $\omega=10^{3} \mathrm{~s}^{-1}$ around the axis that is perpendicular to the accretion disk. The diameter of the perpendicular cut of the toroidal bulk of matter is about $d \sim 20 \mathrm{~km}$ and the distance from the center of this cut to the center of the neutron star is also about $D \sim 20 \mathrm{~km}$. The transversal velocity of matter can be estimated accordingly $v_{\perp}=\omega D=0.067$ that corresponds to $\gamma_{n}=1.002$.

Suppose that the direction of the neutrino propagation is characterized by $\sin \alpha \sim \frac{1}{2}$. If there is a magnetic field $B$ perpendicular to the accretion disk then there is the longitudinal field in respect to the neutrino propagation $B_{\|}=B \sin \alpha \sim \frac{1}{2} B$.

In the straightforward analysis we are particularly interested in the conversion $\nu_{e}^{L} \Leftarrow\left(j_{\perp}\right) \Rightarrow \nu_{e}^{R}$ engendered by interactions with the transversal matter current $\boldsymbol{j}_{\perp}$. Therefore we omit the possible effect of the neutrino magnetic moment interaction with the transversal magnetic field $\mu_{e e} \boldsymbol{B}_{\perp}$ in (42) and get 


$$
E_{\mathrm{eff}}=\left(\frac{\eta}{\gamma}\right)_{e e} \tilde{G} n v_{\perp}=\frac{\cos ^{2} \theta}{\gamma_{11}} \tilde{G} n v_{\perp} \approx \tilde{G} n_{0} \frac{\gamma_{n}}{\gamma_{\nu}} v_{\perp} .
$$

In the considered geometry $\boldsymbol{v} \boldsymbol{\beta}=0$ and for $\Delta_{\text {eff }}$ we use the relation

$$
\begin{aligned}
\Delta_{\text {eff }} & =\left|\left(\frac{\mu}{\gamma}\right)_{e e} \boldsymbol{B}_{\|}+\eta_{e e} \tilde{G} n \boldsymbol{\beta}\right| \\
& =\left|\boldsymbol{B}_{\|}\left(\frac{\mu_{11}}{\gamma_{11}} \cos ^{2} \theta+\frac{\mu_{22}}{\gamma_{22}} \sin ^{2} \theta\right)+\tilde{G} n \boldsymbol{\beta}\right| \\
& \approx\left|\frac{\mu_{11}}{\gamma_{\nu}} B_{\|}-\tilde{G} n_{0} \gamma_{n}\right| .
\end{aligned}
$$

From the demand $E_{\text {eff }}>\Delta_{\text {eff }}$ we get

$$
\left|\frac{\mu_{11} B_{\|}}{\tilde{G} n_{0} \gamma_{n}}-\gamma_{\nu}\right| \leq 1 .
$$

Thus, the criterion (90) is fulfilled if the longitudinal magnetic field is

$$
B_{\|}=B_{\|}^{\mathrm{cr}} \sim \gamma_{n} \gamma_{\nu} \frac{\tilde{G} n_{0}}{\mu_{11}} .
$$

From the obtained estimation of the critical strength $B_{\|}^{\text {cr }}$ of the longitudinal magnetic field $B_{\|}$follows its important dependence on the matter density $n_{0}$. If one takes an estimation for the neutrino magnetic moment $\mu_{11} \sim 3 \times 10^{-11} \mu_{B}$ and $\gamma_{\nu}=2 \times 10^{7}$ then in the case of very low matter density $n_{0} \sim 10^{23} \mathrm{~cm}^{-3}$ the critical field is $B_{\|}^{\text {cr }} \sim 8 \times 10^{-3} B_{0}$, where $B_{0}=\frac{m_{e}^{2}}{e_{0}}=4.41 \times 10^{13}$ Gauss. For higher densities $n_{0} \sim 10^{36} \mathrm{~cm}^{-3}$ we get $B_{\|}^{\text {cr }} \sim 10^{24}$ Gauss that is of the order of the critical field strength $B_{W}=\frac{m_{W}^{2}}{e_{0}}$ where a component of the W-field becomes tachyonic (see [49] and references therein).

From these estimations it would seem that in order to get a reasonable reduction of the term $\tilde{G} n(1-\boldsymbol{v} \boldsymbol{\beta})$ in $\Delta_{\text {eff }}$ given by (42) due to the contribution of $\left(\frac{\mu}{\gamma}\right)_{e e} \boldsymbol{B}_{\|}$the matter density should not be too high to avoid a demand for extremely strong magnetic fields. However, one should also consider the scale of the effective oscillation length whose value in the case of the resonance $\Delta_{\text {eff }} \rightarrow 0$ is given by (45). Even for the case $n_{0} \sim 10^{36} \mathrm{~cm}^{-3}$ the effective length is still extremely large $L_{\text {eff }} \sim 300 \mathrm{~km}$, which makes a hypothetically interesting possibility of the resonant amplification of spin oscillations due to the longitudinal component of the magnetic field unattainable.

\section{NEUTRINO SPIN-FLAVOR OSCILLATIONS $\nu_{e}^{L} \Leftarrow\left(j_{\perp}\right) \Rightarrow \nu_{\mu}^{R}$ ENGENDERED BY TRANSVERSAL MATTER CURRENT}

Here below we consider another interesting consequence of neutrino standard interaction with the transversal matter current that produces the neutrino spin precession and oscillations accompanying in addition with the change of the neutrino flavor. These effects are similar to the neutrino spin-flavor oscillations in the transversal magnetic field which, as it was shown in [24,25], can be amplified by the resonance in the presence of matter.

For the considered case of $\nu_{e}^{L} \Leftarrow\left(j_{\perp B_{\perp}}\right) \Rightarrow \nu_{\mu}^{R}$ from (30) and (33) we get the following neutrino evolution equation:

$$
i \frac{d}{d t}\left(\begin{array}{c}
\nu_{e}^{L} \\
\nu_{\mu}^{R}
\end{array}\right)=\left(\begin{array}{cc}
-\Delta M+\left(\frac{\mu}{\gamma}\right)_{e e} B_{\|}+\tilde{G} n(1-\boldsymbol{v} \boldsymbol{\beta}) & \mu_{e \mu} B_{\perp}+\left(\frac{\eta}{\gamma}\right)_{e \mu} \tilde{G} n v_{\perp} \\
\mu_{e \mu} B_{\perp}+\left(\frac{\eta}{\gamma}\right)_{e \mu} \tilde{G} n v_{\perp} & \Delta M-\left(\frac{\mu}{\gamma}\right)_{\mu \mu} B_{\|}-\tilde{G} n(1-\boldsymbol{v} \boldsymbol{\beta})
\end{array}\right)\left(\begin{array}{c}
\nu_{e}^{L} \\
\nu_{\mu}^{R}
\end{array}\right),
$$

where

$$
\Delta M=\frac{\Delta m^{2} \cos 2 \theta}{4 p_{0}^{\nu}},
$$

and $p_{0}^{\nu}$ is neutrino energy. For the oscillation $\nu_{e}^{L} \Leftarrow\left(j_{\perp}\right) \Rightarrow \nu_{\mu}^{R}$ probability we get

$$
\begin{aligned}
P_{\nu_{e}^{L} \rightarrow \nu_{\mu}^{R}}(x) & =\sin ^{2} 2 \theta_{\mathrm{eff}} \sin ^{2} \frac{\pi x}{L_{\mathrm{eff}}}, \\
\sin ^{2} 2 \theta_{\mathrm{eff}} & =\frac{E_{\mathrm{eff}}^{2}}{E_{\mathrm{eff}}^{2}+\Delta_{\mathrm{eff}}^{2}}, \\
L_{\mathrm{eff}} & =\frac{\pi}{\sqrt{E_{\mathrm{eff}}^{2}+\Delta_{\mathrm{eff}}^{2}}},
\end{aligned}
$$

where

$$
\begin{aligned}
& E_{\text {eff }}=\left|\mu_{e \mu} B_{\perp}+\left(\frac{\eta}{\gamma}\right)_{e \mu} \tilde{G} n v_{\perp}\right|, \\
& \Delta_{\text {eff }}=\left|\Delta M-\frac{1}{2}\left(\frac{\mu_{11}}{\gamma_{11}}+\frac{\mu_{22}}{\gamma_{22}}\right) B_{\|}-\tilde{G} n(1-v \boldsymbol{\beta})\right| .
\end{aligned}
$$

From (57) it follows that the value of $\Delta_{\text {eff }}$ can be diminished by both the neutrino interaction with the longitudinal magnetic field and also by the effect of interaction with matter. 


\section{RESONANCE AMPLIFICATION OF NEUTRINO SPIN-FLAVOR OSCILLATIONS$$
\nu_{e}^{L} \Leftarrow\left(j_{\perp}\right) \Rightarrow \nu_{\mu}^{R}
$$

We are again interested in the situation when the amplitude of oscillations $\sin ^{2} 2 \theta_{\text {eff }}$ in (56) is not small and we use the criterion based on the demand that

$$
\sin ^{2} 2 \theta_{\mathrm{eff}}=\frac{E_{\mathrm{eff}}^{2}}{E_{\mathrm{eff}}^{2}+\Delta_{\mathrm{eff}}^{2}} \geq \frac{1}{2},
$$

which is provided by the condition $E_{\text {eff }} \geq \Delta_{\text {eff }}$. Thus we get the condition

$$
\begin{aligned}
& \left|\mu_{e \mu} B_{\perp}+\left(\frac{\eta}{\gamma}\right)_{e \mu} \tilde{G} n v_{\perp}\right| \\
& \quad \geq\left|\Delta M-\frac{1}{2}\left(\frac{\mu_{11}}{\gamma_{11}}+\frac{\mu_{22}}{\gamma_{22}}\right) B_{\|}-\tilde{G} n(1-\boldsymbol{v} \boldsymbol{\beta})\right| .
\end{aligned}
$$

Consider the case when the effect of the magnetic field is negligible, thus we get

$$
\left|\left(\frac{\eta}{\gamma}\right)_{e \mu} \tilde{G} n v_{\perp}\right| \geq|\Delta M-\tilde{G} n(1-\boldsymbol{v} \boldsymbol{\beta})| .
$$

The effective oscillation length reads

$$
L_{\mathrm{eff}}=\frac{\pi}{\left(\frac{\eta}{\gamma}\right)_{e \mu} \tilde{G} n v_{\perp}} .
$$

In the further evaluations we use the approximation

$$
\left(\frac{\eta}{\gamma}\right)_{e \mu} \approx \frac{\sin 2 \theta}{\gamma_{\nu}}
$$

and get the resonance condition in the form

$$
\frac{\tilde{G} n v_{\perp}}{\gamma_{\nu}} \sin 2 \theta+\tilde{G} n(1-\boldsymbol{v} \boldsymbol{\beta}) \geq \Delta M
$$

In the case $v_{\|}=0$ we get

$$
\frac{\tilde{G} n v_{\perp}}{\gamma_{\nu}} \sin 2 \theta+\tilde{G} n \approx \tilde{G} n .
$$

Finally, the criterion (58) is fulfilled when the following condition is valid:

$$
\tilde{G} n \geq \Delta M .
$$

Consider again an environment peculiar to models of short gamma-ray bursts discussed in Sec. IV B (see also $[47,48])$. The mass squared difference and mixing angle are taken from the solar neutrino measurements, $\Delta m^{2}=$ $7.37 \times 10^{-5} \mathrm{eV}^{2}, \quad \sin ^{2} \theta=0.297 \quad(\cos 2 \theta=0.406) \quad[50]$.
Consider neutrino with energy $p_{0}^{\nu}=10^{6} \mathrm{eV}$ and moving matter characterized by $\gamma_{n}=1.002$. Thus, we get

$$
\Delta M=0.75 \times 10^{-11} \mathrm{eV} .
$$

Accounting for the estimation

$$
\tilde{G}=\frac{G_{F}}{2 \sqrt{2}}=0.4 \times 10^{-23} \mathrm{eV}^{-2},
$$

from the criterion (65) we get the quite reasonable condition on the density of neutrons,

$$
n_{0} \geq \frac{\Delta M}{\tilde{G}}=10^{12} \mathrm{eV}^{3} \approx 10^{26} \mathrm{~cm}^{-3} .
$$

The corresponding oscillation length is approximately

$$
L_{\mathrm{eff}}=\frac{\pi}{\left(\frac{\eta}{\gamma}\right)_{e \mu} \tilde{G} n v_{\perp}} \approx 5 \times 10^{11} \mathrm{~km} .
$$

The oscillation length can be within the scale of short gamma-ray bursts discussed in Sec. IV B:

$$
L_{\mathrm{eff}} \approx 10 \mathrm{~km}
$$

if the matter density equals $n_{0} \approx 5 \times 10^{36} \mathrm{~cm}^{-3}$.

\section{NEUTRINO SPIN-FLAVOR OSCILLATIONS $\nu_{e}^{L} \Leftarrow\left(j_{\perp}\right) \Rightarrow \nu_{\mu}^{R}$ ENGENDERED BY NONSTANDARD INTERACTIONS}

Quite recently [51] it has been shown that nonstandard interactions (NSI) of neutrinos with matter [52-54] can significantly alter neutrino flavor evolution in supernovae with the potential to impact explosion dynamics, nucleosynthesis, and the neutrinos signal.

Obviously, neutrino spin oscillations can be also engendered by NSI of neutrinos with the transversal matter currents.

The effect of nonstandard interactions is usually parametrized by a set of matrices that introduce new contributions to the matter potential of neutrinos. The strength of this new potential is dependent on constituent of the matter and can also contain off-diagonal contributions known as flavor changing neutral currents. Thus, the neutrino spinflavor oscillations, for instance $\nu_{e}^{L} \Leftarrow\left(j_{\perp}\right) \Rightarrow \nu_{\mu}^{R}$, can be engendered by the nonstandard interactions with the transversal matter current.

From the Lagrangian of NSI [52-54]

$$
\begin{aligned}
-L_{N S I}^{\mathrm{eff}} & =\varepsilon_{\alpha \beta}^{f P} 2 \sqrt{2} G_{F}\left(\bar{\nu}_{\alpha} \gamma_{\rho} L \nu_{\beta}\right)\left(\bar{f} \gamma^{\rho} P f\right), \\
L, R & =\left(1 \pm \gamma^{5}\right) / 2
\end{aligned}
$$

(where $f=u, d, e$ and $\alpha, \beta=e, \mu$ ) for the medium with only neutrons, one gets 


$$
\begin{aligned}
-L_{N S I}^{\mathrm{eff}} & =-f^{\mu} \sum_{\alpha, \beta}\left(\varepsilon_{\alpha \beta}^{u L}+2 \varepsilon_{\alpha \beta}^{d L}\right) \bar{\nu}_{\alpha}(x) \gamma_{\mu} \frac{1+\gamma_{5}}{2} \nu_{\beta}(x) \\
& =-f^{\mu} \sum_{\alpha, \beta} \varepsilon_{\alpha \beta}^{n} \bar{\nu}_{\alpha}(x) \gamma_{\mu} \frac{1+\gamma_{5}}{2} \nu_{\beta}(x),
\end{aligned}
$$

where the notation is introduced:

$$
\varepsilon_{\alpha \beta}^{n}=\varepsilon_{\alpha \beta}^{u L}+2 \varepsilon_{\alpha \beta}^{d L} .
$$

The neutrino evolution equation with standard and nonstandard interactions in the flavor basis is

$$
i \frac{d}{d t} \nu_{f}=\left(H_{0}+\Delta H_{\mathrm{SM}}+\Delta H_{N S I}\right) \nu_{f} .
$$

Accounting for the neutrino mixing, contributions to the sum (72) can be expressed in terms of the neutrino mass states. For the characteristic contributions in (72) we get the following expressions $\left(\Gamma_{\mu}=\gamma_{\mu} \frac{1+\gamma_{5}}{2}\right)$ :

$$
\begin{aligned}
\varepsilon_{e e}^{n} \bar{\nu}_{e}^{s} \Gamma^{\lambda} \nu_{e}^{s^{\prime}}= & \varepsilon_{e e}^{n}\left(\bar{\nu}_{1}^{s} \Gamma^{\lambda} \nu_{1}^{s^{\prime}} \cos ^{2} \theta \bar{\nu}_{2}^{s} \Gamma^{\lambda} \nu_{2}^{s^{\prime}} \sin ^{2} \theta\right. \\
& \left.+\frac{1}{2}\left(\bar{\nu}_{1}^{s} \Gamma^{\lambda} \nu_{2}^{s^{\prime}}+\bar{\nu}_{2}^{s} \Gamma^{\lambda} \nu_{1}^{s^{\prime}}\right) \sin 2 \theta\right), \\
\varepsilon_{\mu \mu}^{n} \bar{\nu}_{\mu}^{s} \Gamma^{\lambda} \nu_{\mu}^{s^{\prime}}= & \varepsilon_{\mu \mu}^{n}\left(\bar{\nu}_{1}^{s} \Gamma^{\lambda} \nu_{1}^{s^{\prime}} \sin ^{2} \theta+\bar{\nu}_{2}^{s} \Gamma^{\lambda} \nu_{2}^{s^{\prime}} \cos ^{2} \theta\right. \\
& \left.-\frac{1}{2}\left(\bar{\nu}_{1}^{s} \Gamma^{\lambda} \nu_{2}^{s^{\prime}}+\bar{\nu}_{2}^{s} \Gamma^{\lambda} \nu_{1}^{s^{\prime}}\right) \sin 2 \theta\right), \\
\varepsilon_{e \mu}^{n} \bar{\nu}_{e}^{s} \Gamma^{\lambda} \nu_{\mu}^{s^{\prime}}= & \varepsilon_{e \mu}^{n}\left(-\bar{\nu}_{2}^{s} \Gamma^{\lambda} \nu_{1}^{s^{\prime}} \sin ^{2} \theta+\bar{\nu}_{1}^{s} \Gamma^{\lambda} \nu_{2}^{s^{\prime}} \cos ^{2} \theta\right. \\
& \left.-\frac{1}{2}\left(\bar{\nu}_{1}^{s} \Gamma^{\lambda} \nu_{1}^{s^{\prime}}-\bar{\nu}_{2}^{s} \Gamma^{\lambda} \nu_{2}^{s^{\prime}}\right) \sin 2 \theta\right),
\end{aligned}
$$

$$
\begin{aligned}
\varepsilon_{\mu e}^{n} \bar{\nu}_{\mu}^{s} \Gamma^{\lambda} \nu_{e}^{s^{\prime}}= & \varepsilon_{\mu e}^{n}\left(\bar{\nu}_{2}^{s} \Gamma^{\lambda} \nu_{1}^{s^{\prime}} \cos ^{2} \theta-\bar{\nu}_{1}^{s} \Gamma^{\lambda} \nu_{2}^{s^{\prime}} \sin ^{2} \theta\right. \\
& \left.-\frac{1}{2}\left(\bar{\nu}_{1}^{s} \Gamma^{\lambda} \nu_{1}^{s^{\prime}}-\bar{\nu}_{2}^{s} \Gamma^{\lambda} \nu_{2}^{s^{\prime}}\right) \sin 2 \theta\right) .
\end{aligned}
$$

The matrix elements of the NSI contribution to the evolution Hamiltonian can be calculated accounting for (75)-(78) and, as it is described in Sec. III B, in the neutrino mass basis using the vacuum neutrino wave functions given by (19). Note that the evolution equation in the neutrino mass basis, accounting only for the NSI, is

$$
i \frac{d}{d t} \nu_{\alpha}^{s}=\left(H_{0}^{m}+\Delta H_{N S I}^{m}\right) \nu_{\alpha}^{s} .
$$

Here $H_{0}^{m}$ is the neutrino evolution Hamiltonian for the mass states in vacuum, $\alpha=1,2$ and $s= \pm 1$, and

$$
\Delta H_{N S I}^{m}=\tilde{G} n \times\left(\begin{array}{cccc}
0 & \frac{v_{\perp}}{\gamma_{11}} e_{11} & 0 & \frac{v_{\perp}}{\gamma_{11}} e_{12} \\
\frac{v_{\perp}}{\gamma_{11}} e_{11} & 2\left(1-v_{\|}\right) e_{11} & \frac{v_{\perp}}{\gamma_{22}} e_{12} & 2\left(1-v_{\|}\right) e_{12} \\
0 & \frac{v_{\perp}}{\gamma_{22}} e_{12} & 0 & \frac{v_{\perp}}{\gamma_{22}} e_{22} \\
\frac{v_{\perp}}{\gamma_{11}} e_{12} & 2\left(1-v_{\|}\right) e_{12} & \frac{v_{\perp}}{\gamma_{22}} e_{22} & 2\left(1-v_{\|}\right) e_{22}
\end{array}\right) \text {, }
$$

where

$$
\begin{aligned}
& e_{11}=\varepsilon_{e e}^{n} \cos ^{2} \theta+\varepsilon_{\mu \mu}^{n} \sin ^{2} \theta-\varepsilon_{e \mu}^{n} \sin 2 \theta, \\
& e_{12}=\frac{1}{2}\left(\varepsilon_{e e}^{n}-\varepsilon_{\mu \mu}^{n}\right) \sin 2 \theta+\varepsilon_{e \mu}^{n} \cos 2 \theta, \\
& e_{22}=\varepsilon_{e e}^{n} \sin ^{2} \theta+\varepsilon_{\mu \mu}^{n} \cos ^{2} \theta+\varepsilon_{e \mu}^{n} \sin 2 \theta .
\end{aligned}
$$

Summing up three contributions to the neutrino Hamiltonian in (79) for the neutrino spin-flavor oscillations $\nu_{e}^{L} \Leftarrow\left(j_{\perp}\right) \Rightarrow \nu_{\mu}^{R}$, we get

$$
i \frac{d}{d t}\left(\begin{array}{c}
\nu_{e}^{L} \\
\nu_{\mu}^{R}
\end{array}\right)=\left(\begin{array}{cc}
-\Delta M+\left(\frac{\mu}{\gamma}\right)_{e e} B_{\|}+2 \tilde{G} n(1-\boldsymbol{v} \boldsymbol{\beta})\left(1+\tilde{\varepsilon}_{e e}\right) & \mu_{e \mu} B_{\perp}+\left(\frac{\eta}{\gamma}\right)_{e \mu} \tilde{G} n v_{\perp}\left(1+\tilde{\varepsilon}_{e \mu}\right) \\
\mu_{e \mu} B_{\perp}+\left(\frac{\eta}{\gamma}\right)_{e \mu} \tilde{G} n v_{\perp}\left(1+\tilde{\varepsilon}_{e \mu}\right) & \Delta M-\left(\frac{\mu}{\gamma}\right)_{\mu \mu} B_{\|}
\end{array}\right)\left(\begin{array}{c}
\nu_{e}^{L} \\
\nu_{\mu}^{R}
\end{array}\right),
$$

where

$\tilde{\varepsilon}_{e e}=h_{22} \cos ^{2} \theta+h_{44} \sin ^{2} \theta+h_{24} \sin 2 \theta$,

$\tilde{\varepsilon}_{e \mu}=-\frac{h_{14}}{\gamma_{11}} \sin ^{2} \theta+\frac{h_{23}}{\gamma_{22}} \cos ^{2} \theta+\frac{1}{2}\left(\frac{h_{34}}{\gamma_{22}}-\frac{h_{12}}{\gamma_{11}}\right) \sin 2 \theta$.

Note that in the neutrino spin-flavor evolution equation (84) the standard and nonstandard interactions with matter and also possible influence of the magnetic field are accounted for.

For the oscillation $\nu_{e}^{L} \Leftarrow\left(j_{\perp}\right) \Rightarrow \nu_{\mu}^{R}$ probability in the considered case we get

$$
\begin{aligned}
P_{\nu_{e}^{L} \rightarrow \nu_{e}^{R}}(x) & =\sin ^{2} 2 \theta_{\mathrm{eff}} \sin ^{2} \frac{\pi x}{L_{\mathrm{eff}}}, \\
L_{\mathrm{eff}} & =\frac{\pi}{\sqrt{E_{\mathrm{eff}}^{2}+\Delta_{\mathrm{eff}}^{2}}},
\end{aligned}
$$


where

$$
E_{\text {eff }}=\left|\mu_{e \mu} B_{\perp}+\left(\frac{\eta}{\gamma}\right)_{e \mu} \tilde{G} n v_{\perp}\left(1+\tilde{\varepsilon}_{e \mu}\right)\right|,
$$

and

$\Delta_{\mathrm{eff}}=\left|\Delta M-\frac{1}{2}\left(\left(\frac{\mu}{\gamma}\right)_{e e}+\left(\frac{\mu}{\gamma}\right)_{\mu \mu}\right) B_{\|}-\tilde{G} n(1-\boldsymbol{v} \boldsymbol{\beta})\left(1+\tilde{\varepsilon}_{e e}\right)\right|$.

The amplitude of oscillations $\sin ^{2} 2 \theta_{\text {eff }}$ in (87) is not small, when

$$
\sin ^{2} 2 \theta_{\mathrm{eff}}=\frac{E_{\mathrm{eff}}^{2}}{E_{\mathrm{eff}}^{2}+\Delta_{\mathrm{eff}}^{2}} \geq \frac{1}{2}
$$

Thus, we arrive to the condition

$$
\begin{aligned}
& \left|\mu_{e \mu} B_{\perp}+\left(\frac{\eta}{\gamma}\right)_{e \mu} \tilde{G} n v_{\perp}\left(1+\tilde{\varepsilon}_{e \mu}\right)\right| \\
& \geq\left|\Delta M-\frac{1}{2}\left(\left(\frac{\mu}{\gamma}\right)_{e e}+\left(\frac{\mu}{\gamma}\right)_{\mu \mu}\right) B_{\|}-\tilde{G} n(1-v \boldsymbol{\beta})\left(1+\tilde{\varepsilon}_{e e}\right)\right| .
\end{aligned}
$$

We are in particular interested in the effect of the NSI, thus neglecting the influence of the magnetic field we get

$$
\left|\left(\frac{\eta}{\gamma}\right)_{e \mu} \tilde{G} n v_{\perp}\left(1+\tilde{\varepsilon}_{e \mu}\right)\right| \geq\left|\Delta M-\tilde{G} n(1-\boldsymbol{v} \boldsymbol{\beta})\left(1+\tilde{\varepsilon}_{e e}\right)\right| .
$$

Using the approximation

$$
\left(\frac{\eta}{\gamma}\right)_{e \mu} \approx \frac{\sin 2 \theta}{\gamma_{\nu}}
$$

and for the resonance condition for $\nu_{e}^{L} \Leftarrow\left(j_{\perp}\right) \Rightarrow \nu_{\mu}^{R}$ we get

$$
\frac{\tilde{G} n v_{\perp}}{\gamma_{\nu}}\left(1+\tilde{\varepsilon}_{e \mu}\right) \sin 2 \theta+\tilde{G} n(1-\boldsymbol{v} \boldsymbol{\beta})\left(1+\tilde{\varepsilon}_{e e}\right) \geq \Delta M .
$$

In the case $v_{\|}=0$,

$$
\frac{\tilde{G} n v_{\perp}}{\gamma_{\nu}}\left(1+\tilde{\varepsilon}_{e \mu}\right) \sin 2 \theta+\tilde{G} n\left(1+\tilde{\varepsilon}_{e e}\right) \approx \tilde{G} n\left(1+\tilde{\varepsilon}_{e e}\right),
$$

and from (94) we get

$$
n \geq \frac{\Delta M}{\tilde{G}\left(1+\tilde{\varepsilon}_{e e}\right)} .
$$

For definiteness we use in our estimations the values of nonstandard parameters from [53]:

$$
\begin{aligned}
& \varepsilon_{e e}^{u L}=\varepsilon_{e e}^{d L}=0.3 \\
& \varepsilon_{\mu \mu}^{u L}=\varepsilon_{\mu \mu}^{d L}=0.005 \\
& \varepsilon_{e \mu}^{u L}=\varepsilon_{e \mu}^{d L}=0.023
\end{aligned}
$$

thus

$$
\tilde{\varepsilon}_{e e}=0.6
$$

Finally, for the mass squared difference and mixing angle taken from the solar neutrino measurements, $\Delta m^{2}=$ $7.37 \times 10^{-5} \mathrm{eV}^{2}, \sin ^{2} \theta=0.297$, the neutrino energy $p_{0}^{\nu}=$ $10^{6} \mathrm{eV}$ and moving matter characterized by $\gamma_{n}=1.002$ for the matter density we get

$$
n_{0} \geq 0.625 \times 10^{26} \mathrm{~cm}^{-3} \text {. }
$$

The conclusion is that the account for the NSI can soften the demand on the density of the transversal matter current needed for the resonance amplification of the neutrino spinflavor oscillations $\nu_{e}^{L} \Leftarrow\left(j_{\perp}\right) \Rightarrow \nu_{\mu}^{R}$.

\section{CONCLUSIONS}

In this paper we develop the quantum treatment of the effect of the neutrino spin $\nu_{e}^{L} \Leftarrow\left(j_{\perp}\right) \Rightarrow \nu_{e}^{R}$ and spin-flavor $\nu_{e}^{L} \Leftarrow\left(j_{\perp}\right) \Rightarrow \nu_{\mu}^{R}$ oscillations engendered by the transversal matter current that was predicted in [2] on the basis of the semiclassical treatment of the neutrino spin evolution in the background matter. For definiteness, matter composed of neutrons is considered.

Several particular cases of the neutrino spin oscillations resonance amplifications are considered. It is shown, in particular, that the resonance in the probability of the neutrino spin oscillations $\nu_{e}^{L} \Leftarrow\left(j_{\perp}\right) \Rightarrow \nu_{e}^{R}$ can be produced by the longitudinal component of the ultrarelativistic background matter current with $\gamma_{n} \geq \gamma_{\nu}{ }^{1 / 2} \sim 3 \times 10^{3}$.

The resonance amplification of the probability of the neutrino spin oscillations $\nu_{e}^{L} \Leftarrow\left(j_{\perp}\right) \Rightarrow \nu_{e}^{R}$ by the longitudinal magnetic field has been also considered for an astrophysical environment that can be realized by models of short gamma-ray bursts (sGRB) (see [47,48]).

The novel effect of the neutrino spin-flavor oscillations $\nu_{e}^{L} \Leftarrow\left(j_{\perp}\right) \Rightarrow \nu_{\mu}^{R}$ engendered by the transversal matter current have been also considered and the resonance amplification of its probability has been considered for different values of the matter density $n_{0} \sim 10^{26}-10^{37} \mathrm{~cm}^{-3}$.

We also consider for the first time the neutrino spinflavor oscillations $\nu_{e}^{L} \Leftarrow\left(j_{\perp}\right) \Rightarrow \nu_{\mu}^{R}$ engendered by the 
transversal matter current with the nonstandard interactions. The oscillation probability has been derived and an estimation for the resonance matter density has been obtained with use of the realistic strengths of the neutrino nonstandard interactions.

It is supposed throughout the paper that neutrino oscillations proceed under the validity of the adiabaticity condition, i.e., under the influence of constant or slowly varying magnetic fields and matter current densities. The opposite case (nonadiabatic regime in neutrino oscillations) requires special treatment.

For the general neutrino evolution equation,

$$
i \frac{d}{d t}\left(\begin{array}{c}
\nu_{i} \\
\nu_{j}
\end{array}\right)=\left(\begin{array}{ll}
H_{i i} & H_{i j} \\
H_{j i} & H_{j j}
\end{array}\right)\left(\begin{array}{c}
\nu_{i} \\
\nu_{j}
\end{array}\right)
$$

the adiabaticity condition can be represented (see [30]) in the form

$$
\begin{aligned}
& \left|\left(H_{j j}-H_{i i}\right) \frac{\partial}{\partial x}\left(H_{i j}+H_{j i}\right)-\left(H_{i j}+H_{j i}\right) \frac{\partial}{\partial x}\left(H_{j j}-H_{i i}\right)\right| \\
& \ll\left[\left(H_{j j}-H_{i i}\right)^{2}+\left(H_{i j}+H_{j i}\right)^{2}\right]^{\frac{3}{2}} .
\end{aligned}
$$

This condition can be rewritten in a more compact form,

$$
\left|\Delta_{\text {eff }} \frac{\partial E_{\text {eff }}}{\partial x}-E_{\text {eff }} \frac{\partial \Delta_{\text {eff }}}{\partial x}\right| \ll 4\left[\Delta_{\text {eff }}^{2}+E_{\text {eff }}^{2}\right]^{\frac{3}{2}},
$$

which, with the appropriate choice of $E_{\text {eff }}$ and $\Delta_{\text {eff }}$ values, can be used to analyze the situation in several specific cases discussed above. The Eq. (100) clearly shows that the adiabaticity condition imposes restrictions on changes in the strength of a magnetic field, matter density and velocity along the direction of the neutrino propagation.

Consider, in particular, neutrino spin-flavor oscillations $\nu_{e}^{L} \Leftarrow\left(j_{\perp}\right) \Rightarrow \nu_{\mu}^{R}$ in an environment peculiar to models of short gamma-ray bursts $[47,48]$ discussed in Sec. IV B. In accordance with the model in question, the matter density of a rotating bulk may vary in the range from $\sim 10^{38} \mathrm{~cm}^{3}$ to $\sim 10^{33} \mathrm{~cm}^{3}$ at the characteristic distance of about $20 \mathrm{~km}$. Accounting to the linear dependence the matter velocity $v$ on the neutrino traveled distance in a rotating environment and following the discussion on validity of the adiabaticity condition in case of a magnetized neutron star [30], we conclude that this condition is fulfilled along the most neutrino travel distance. The adiabaticity condition can most probably be violated in a quite narrow outer layer of the rotating bulk $\delta x \ll d$ which in turn is much thinner the oscillation length $L_{\text {eff }}$ given by (70). Note that in general the origin and strengths of compact astrophysical objects magnetic fields are quite controversial and there are various models of magnetic fields discussed in the literature (see, for instance [55-57]).

The developed quantum theory of the neutrino spin $\nu_{e}^{L} \Leftarrow\left(j_{\perp}\right) \Rightarrow \nu_{e}^{R}$ and spin-flavor $\nu_{e}^{L} \Leftarrow\left(j_{\perp}\right) \Rightarrow \nu_{\mu}^{R}$ oscillations engendered by the transversal matter current and the performed studies of different possibilities for the resonance amplification of the oscillations probabilities provide the conclusion that these phenomena might have important consequences for generation and propagation of neutrino fluxes in extreme astrophysical interments, in particular, peculiar for supernovae. In the performed above studies we consider Dirac neutrinos, however the case of Majorana neutrinos can be treated in a quite similar way. In this concern, it is interesting to recall the statement $[58,59]$ that future high-precision observations of supernova fluxes, for instance, in the JUNO experiment [60], may reveal the effect of the resonate amplification of Majorana neutrino spin-flavor oscillations in magnetic fields due to the neutrino collective effect. From the performed above studies it follows that the neutrino spin-flavor oscillations engendered by interactions with the transversal environment matter current, including possible neutrino selfinteractions, can provide much more important effect on the observed neutrino currents in future large-volume scintillator detectors than the corresponding effect from the neutrino transition moment interaction with magnetic fields.

\section{ACKNOWLEDGMENTS}

The authors are thankful to Konstantin Kouzakov, Artem Popov and Konstantin Stankevich for useful discussions. This work is supported by the Russian Basic Research Foundation Grants No. 16-02-01023 and No. 17-52-53133.
[1] B. Pontecorvo, Mesonium and anti-mesonium, Zh. Eksp. Teor. Fiz. 33, 549 (1957) [Sov. Phys. JETP 6, 429 (1957)].

[2] A. Studenikin, Neutrinos in electromagnetic fields and moving media, Phys. At. Nucl. 67, 993 (2004).

[3] A. Studenikin, The four new effects in neutrino oscillations, Nucl. Phys. B, Proc. Suppl. 143, 570 (2005); Frascati Phys. Ser. 34, 155 (2004).
[4] V. Cirigliano, G. Fuller, and A. Vlasenko, A new spin on neutrino quantum kinetics, Phys. Lett. B 747, 27 (2015).

[5] C. Volpe, Neutrino quantum kinetic equations, Int. J. Mod. Phys. E 24, 1541009 (2015).

[6] A. Kartavtsev, G. Raffelt, and H. Vogel, Neutrino propagation in media: Flavor, helicity, and pair correlations, Phys. Rev. D 91, 125020 (2015). 
[7] A. Dobrynina, A. Kartavtsev, and G. Raffelt, Helicity oscillations of Dirac and Majorana neutrinos, Phys. Rev. D 93, 125030 (2016).

[8] J. Y. Tian, A. V. Patwardhan, and G. M. Fuller, Prospects for neutrino spin coherence in supernovae, Phys. Rev. D 95, 063004 (2017).

[9] B. Pontecorvo, Inverse beta processes and nonconservation of lepton charge, Zh. Eksp. Teor. Fiz. 34, 247 (1957) [Sov. Phys. JETP 7, 172 (1958)]; JINR Report No. P-45, Dubna, 1957.

[10] S. Bilenky, Neutrino oscillations: From a historical perspective to the present status, Nucl. Phys. B908, 2 (2016).

[11] Z. Maki, M. Nakagawa, and S. Sakata, Remarks on the unified model of elementary particles, Prog. Theor. Phys. 28, 870 (1962).

[12] V. N. Gribov and B. Pontecorvo, Neutrino astronomy and lepton charge, Phys. Lett. 28B, 493 (1969).

[13] S. M. Bilenky and B. Pontecorvo, Quark-lepton analogy and neutrino oscillations, Phys. Lett. 61B, 248 (1976).

[14] L. Wolfenstein, Neutrino oscillations in matter, Phys. Rev. D 17, 2369 (1978).

[15] S. P. Mikheev and A. Y. Smirnov, Resonance amplification of oscillations in matter and spectroscopy of solar neutrinos, Yad. Fiz. 42, 1441 (1985) [Sov. J. Nucl. Phys. 42, 913 (1985)].

[16] K. Fujikawa and R. Shrock, Magnetic Moment of a Massive Neutrino and Neutrino-Spin Rotation, Phys. Rev. Lett. 45, 963 (1980).

[17] A. G. Beda, V. B. Brudanin, V. G. Egorov, D. V. Medvedev, V. S. Pogosov, M. V. Shirchenko, and A. S. Starostin, The results of search for the neutrino magnetic moment in GEMMA experiment, Adv. High Energy Phys. 2012, 350150 (2012).

[18] G. Raffelt, New Bound on Neutrino Dipole Moments from Globular-Cluster Stars, Phys. Rev. Lett. 64, 2856 (1990).

[19] C. Giunti and A. Studenikin, Neutrino electromagnetic interactions: A window to new physics, Rev. Mod. Phys. 87, 531 (2015).

[20] A. Studenikin, Neutrino electromagnetic properties: a window to new physics-II, Proc. Sci., EPS-HEP2017 (2017) 137 [arXiv:1801.08887].

[21] A. Cisneros, Effect of neutrino magnetic moment on solar neutrino observations, Astrophys. Space Sci. 10, 87 (1971).

[22] J. Schechter and J. W. F. Valle, Majorana neutrinos and magnetic fields, Phys. Rev. D 24, 1883 (1981).

[23] L. Okun, M. Voloshin, and M. Vysotsky, Electromagnetic properties of neutrino and possible semiannual variation cycle of the solar neutrino flux, Yad. Fiz. 44, 677 (1986) [Sov. J. Nucl. Phys. 44, 440 (1986)].

[24] E. Akhmedov, Resonant amplification of neutrino spin rotation in matter and the solar-neutrino problem, Phys. Lett. B 213, 64 (1988).

[25] C. S. Lim and W. J. Marciano, Resonant spin-flavor precession of solar and supernova neutrinos, Phys. Rev. D 37, 1368 (1988).

[26] E. Akhmedov and M. Khlopov, Resonant amplification of neutrino oscillations in longitudinal magnetic field, Mod. Phys. Lett. A 03, 451 (1988).

[27] J. Vidal and J. Wudka, Non-dynamical contributions to leftright transitions in the solar neutrino problem, Phys. Lett. B 249, 473 (1990).
[28] A. Y. Smirnov, The geometrical phase in neutrino spin precession and the solar neutrino problem, Phys. Lett. B 260, 161 (1991).

[29] E. K. Akhmedov, S. T. Petcov, and A. Y. Smirnov, Neutrinos with mixing in twisting magnetic fields, Phys. Rev. D 48, 2167 (1993).

[30] G. G. Likhachev and A. I. Studenikin, Neutrino oscillations in the magnetic field of the sun, supernovae, and neutron stars, Zh. Eksp. Teor. Fiz. 108, 769 (1995) [J. Exp. Theor. Phys. 81, 419 (1995)].

[31] M. Dvornikov, Neutrino oscillations in matter and in twisting magnetic fields, J. Phys. G 35, 025003 (2008).

[32] A. Dmitriev, R. Fabbricatore, and A. Studenikin, Neutrino electromagnetic properties: new approach to oscillations in magnetic fields, Proc. Sci., CORFU2014 (2015) 050 [arXiv:1506.05311].

[33] A. Studenikin, Electromagnetic properties of neutrinos: three new phenomena in neutrino spin oscillations, Eur. Phys. J. Web Conf. 125, 04018 (2016).

[34] A. Popov and A. Studenikin, Neutrino oscillations and exact eigenstates in magnetic field, arXiv:1803.05755.

[35] P. Kurashvili, K. A. Kouzakov, L. Chotorlishvili, and A. I. Studenikin, Spin-flavor oscillations of ultrahigh-energy cosmic neutrinos in interstellar space: The role of neutrino magnetic moments, Phys. Rev. D 96, 103017 (2017).

[36] A. Egorov, A. Lobanov, and A. Studenikin, Neutrino oscillations in electromagnetic fields, Phys. Lett. B 491, 137 (2000).

[37] A. Lobanov and A. Studenikin, Neutrino oscillations in moving and polarized matter under the influence of electromagnetic fields, Phys. Lett. B 515, 94 (2001).

[38] M. S. Dvornikov and A. I. Studenikin, Neutrino oscillations in the field of a linearly polarized electromagnetic wave, Yad. Fiz. 64, 1705 (2001) [Phys. At. Nucl. 64, 1624 (2001)].

[39] M. S. Dvornikov and A. I. Studenikin, Parametric resonance in neutrino oscillations in periodically varying electromagnetic fields, Yad. Fiz. 67, 741 (2004) [Phys. At. Nucl. 67, 719 (2004)].

[40] M. Dvornikov and A. Studenikin, Neutrino spin evolution in presence of general external fields, J. High Energy Phys. 09 (2002) 016.

[41] R. Fabbricatore, A. Grigoriev, and A. Studenikin, Neutrino spin-flavor oscillations derived from the mass basis, J. Phys. Conf. Ser. 718, 062058 (2016).

[42] A. Studenikin, Neutrino spin and spin-flavour oscillations in transversally moving or polarized matter, J. Phys. Conf. Ser. 888, 012221 (2017).

[43] A. Studenikin, Status and perspectives of neutrino magnetic moments, J. Phys. Conf. Ser. 718, 062076 (2016).

[44] A. Studenikin, From neutrino electromagnetic interactions to spin oscillations in transversal matter currents, Proc. Sci., NOW2016 (2016) 070 [arXiv:1706.01100].

[45] A.Studenikin, New phenomenon of neutrino spin oscillations in transversal matter currents, Proc. Sci., CORFU2017 (2018), 085.

[46] The typos in (30) and the last formulas of (31) and (32) as they appear in $[33,41]$ are corrected here.

[47] A. Grigoriev, A. Lokhov, A. Studenikin, and A. Ternov, Spin light of neutrino in astrophysical environments, J. Cosmol. Astropart. Phys. 11 (2017) 024. 
[48] A. Perego, S. Rosswog, R. M. Cabezn, O. Korobkin, R. Kppeli, A. Arcones, and M. Liebendrfer, Neutrino-driven winds from neutron star merger remnants, Mon. Not. R. Astron. Soc. 443, 3134 (2014).

[49] N. K. Nielsen, Higgs boson decay into two photons in an electromagnetic background field, Phys. Rev. D 90, 016010 (2014).

[50] C. Patrignani et al. (Particle Data Group), Review of Particle Physics, Chin. Phys. C 40, 100001 (2016).

[51] C. J. Stapleford, D. J. Väänänen, J. P. Kneller, G. C. McLaughlin, and B.T. Shapiro, Nonstandard neutrino interactions in supernovae, Phys. Rev. D 94, 093007 (2016).

[52] T. Ohlsson, Status of non-standard neutrino interactions, Rep. Prog. Phys. 76, 044201 (2013).

[53] O. G. Miranda and H. Nunokawa, Non standard neutrino interactions: current status and future prospects, New J. Phys. 17, 095002 (2015).
[54] Y. Farzan and M. Tortola, Neutrino oscillations and nonstandard interactions, Front. Phys. 6, 10 (2018).

[55] N. V. Ardeljan, G. S. Bisnovatyi-Kogan, and S. G. Moiseenko, Magnetorotational supernovae, Mon. Not. R. Astron. Soc. 359, 333 (2005).

[56] A. Y. Potekhin, J. A. Pons, and D. Page, Neutron starscooling and transport, Space Sci. Rev. 191, 239 (2015).

[57] H. C. Spruit, Origin of neutron star magnetic fields, AIP Conf. Proc. 983, 391 (2008).

[58] A. de Gouvea and S. Shalgar, Effect of transition magnetic moments on collective supernova neutrino oscillations, J. Cosmol. Astropart. Phys. 10 (2012) 027.

[59] A. de Gouvea and S. Shalgar, Transition magnetic moments and collective neutrino oscillations: Three-flavor effects and detectability, J. Cosmol. Astropart. Phys. 04 (2013) 018.

[60] F. An et al. (JUNO Collaboration), Neutrino physics with JUNO, J. Phys. G 43, 030401 (2016). 